\title{
Performance analysis of spectrum sharing mechanisms in cognitive radio networks
}

\author{
Chen Peipei, Zhang Qinyu*, Zhang Yalin and Wang Ye
}

\begin{abstract}
In this article, a non-preemptive (NP) mechanism is proposed to improve the quality-of-service (QoS) of secondary users (SUs) in joint leasing and sensing-based cognitive radio networks (CRNs). In this spectrum-sharing mechanism, a primary user (PU) could not forcibly terminate a SU with ongoing transmission. Both the typical preemptive and the proposed NP mechanisms are modeled by multi-dimensional Markov chains with three state variables. A decomposition-approximated method is used to derive the closed-form solutions of the steady-state probabilities in the Markov chains. The analytical results are verified by numerical results. System parameters that affect performance metrics are also investigated in these two mechanisms. The simulation results show that in the proposed mechanism the performance metrics of SUs such as force-termination probability and mean system delay are improved significantly, with an acceptable loss of PUs' QoS in terms of mean waiting time and blocking probability. A QoS tradeoff can be achieved between the primary and the secondary systems. For QoS improvement of SUs, the proposed NP mechanism outperforms the preemptive mechanism in joint leasing and sensing-based CRNs.
\end{abstract}

Keywords: cognitive radio networks, joint leasing and sensing, non-preemptive mechanism, QoS tradeoff

\section{Introduction}

Cognitive radio (CR) has been considered as a viable technique to improve the utilization of spectral resources in a licensed (primary) system [1]. The secondary users (SUs) in the unlicensed (secondary) system are allowed to opportunistically utilize the spectrum holes that are temporarily unoccupied by primary users (PUs). The key enabler is the $\mathrm{SU}$ with $\mathrm{CR}$ technology, which can sense the spectrum hole and accordingly adjust its transmission parameters. The main idea of CR is that SUs exploit the spectrum holes and take advantage of them opportunistically. Therefore, the spectrum sharing mechanism in CR networks (CRNs) becomes a hot research topic.

According to the literature related to CRNs, previous study on dynamic spectrum access (DSA) can be categorized as sensing-based access model, leasing-based access model, and joint leasing and sensing-based access model. In sensing-based CRNs [2-5], SUs acquire the information of spectrum holes through spectrum sensing and freely access the unoccupied licensed channels, without paying any leasing fees to primary system. The primary system is

\footnotetext{
* Correspondence: zqy@hit.edu.cn

Department of Electronic and Information Engineering, Shenzhen Graduate School, Harbin Institute of Technology, Shenzhen, China
}

ignorant of SUs, and the quality-of-service (QoS) of PUs should be protected by a specific spectrum sharing mechanism. In leasing-based CRNs [6], the secondary system dynamically leases spectrum from primary system and owns exclusive right to access the leased spectrum. However, the spectrum leasing is not performed in real time and the SUs will keep the exclusive right until the lease term expires, which may cause a great QoS degradation to primary system once the PUs' services grow abruptly. The joint leasing and sensing-based CRN proposed in [7] is widely considered to be a viable market option that benefits both the primary and the secondary systems. The primary system can make extra profit via spectrum leasing (unlike in sensing-based CRNs) and SUs have full flexibility in utilizing the spectrum holes (unlike in leasing-based CRNs). SUs pay the primary system the channel leasing fees only for opportunistic access. The joint leasing and sensing-based model enables more flexible integration of DSA in the licensed spectrum via real-time spectrum leasing.

In this article, we study the spectrum-sharing mechanisms in joint leasing and sensing-based CRNs, which benefit both the primary and the secondary systems. The authors in [8] proposed a preemptive spectrum-sharing 
mechanism in joint leasing and sensing-based CRNs. This preemptive mechanism is the same as the traditional spectrum-sharing mechanism in sensing-based CRNs [2-5], which has the basic requirement that the PUs are not affected by the SUs' opportunistic spectrum utilization. A SU has to vacate the channel promptly when a PU returns and handoff to another spectrum hole. When no spectrum hole is available, the SU's ongoing transmission is terminated and the SU is preempted. In the preemptive mechanism, PUs have preemptive priorities over SUs. The preemptive mechanism causes significant forcetermination probability for SUs [2]. That is not only a waste of resources (power and frequency), but also insufferable for SUs, especially for the SUs who lease spectrum for some guarantees of QoS. We originally present a nonpreemptive (NP) spectrum-sharing mechanism, in which PUs have no preemptive priorities over SUs. A PU would wait for a period of time until the completion of the SU's ongoing transmission when no spectrum hole is available. No SU would forcibly be terminated by PUs. A QoS tradeoff will be achieved between the primary and the secondary systems. We focus on the performance analysis of spectrum-sharing mechanisms, which not only gives the evaluation of the spectrum-sharing mechanisms, but also provides a clue for future researches on strategies of primary and secondary systems in joint leasing and sensingbased CRNs.

The interactions between PUs and SUs in spectrum sharing can be modeled by a multi-dimensional Markov chain. For comparison, both the preemptive and the NP mechanisms are modeled based on the Markov process. Markov theory is an effective method to model the spectrum sharing in CR systems [2,3,5]. However, it is always non-trivial to obtain the exact closed-form solutions of the steady-state probabilities. An approximate method introduced by Ghain and Schwartz $[9,10]$ can be used for analyzing the Markov chain and deducing the approximate closed-form solutions of steady-state probabilities since we suppose that the SUs have much shorter average service time than PUs. Performance metrics such as mean system delay and force-termination probability of $\mathrm{SU}$, average waiting time, and blocking probability of $\mathrm{PU}$ are evaluated with the steady-state probabilities in CRNs. The QoS tradeoff relationships between primary and secondary systems are discussed. In addition, the influences of system parameters on performance metrics have also been presented.

This rest of the article is organized as follows. In Section 2, we first present the system model of a joint leasing and sensing-based CRN, and introduce the preemptive and the NP mechanisms based on three-dimensional Markov chains. We then derive the closed-form solutions of the steady-state probabilities in the Markov chains by decomposition approximation. In Section 3, we give the expressions of performance metrics. To verify the analytical solution, simulation results are carried out and the two spectrum-sharing mechanisms are compared and discussed in Section 4. Finally, conclusion is drawn in Section 5 .

\section{System model}

The joint leasing and sensing-based access model can be described as a CRN with three interacting layers [7]: primary system (with PU access point and PUs), spectrum broker, and secondary system (with SU access point and SUs with CR capabilities). The system model is depicted in Figure 1. The primary system divides the licensed spectrum into two parts. One part consists of reserved channels for PUs transmission only, and the other part consists of the shared channels that can be used by SUs opportunistically. The primary system can temporarily lease its spectrum usage rights of the shared channels to secondary system through the spectrum broker, and get payoff from secondary system as SUs opportunistically utilize the shared channels. The spectrum broker can be either a regulatory authority (e.g., FCC in USA, Ofcom in UK) or an authorized third-party. The spectrum broker works as an interaction entity between the primary and the secondary systems [11]. A contract between the primary and the secondary systems has to be made in spectrum broker. The interactions between the primary and the secondary systems in a three-tier CRN can be modeled by a Stackelberg game [12], where the primary system is the leader and secondary system is the follower. The leader announces its own policies (the range of shared channels, spectrum leasing cost), and the secondary system makes its own decisions (the range of leased channels, service tariff) with the knowledge of the leader's decisions. The primary and the secondary systems exchange their information through spectrum broker. For simplicity, we assume that there are one primary system and one secondary system. In this joint leasing and sensing-based three-tier CRN, the spectrum-sharing mechanism has the major influences on the primary and the secondary systems' decisions. The economic factor is not our focus here and will be considered in our future research.

We assume that there are $N$ licensed channels in a primary system, and each of them has identical bandwidth. Among these $N$ channels, $R$ channels are dedicated for PUs, and $N-R$ channels are shared by PUs and SUs. A SU can sense the shared channels by spectrum sensing and access the channel if it is not occupied by a PU. The $\mathrm{PU}$ and the SU arrival processes follow Poisson process with arrival rates $\lambda_{\mathrm{p}}$ and $\lambda_{\mathrm{s}}$, respectively. The service in the CRN is a single-slot first come first served transmission. The service time of the PU follows exponential distribution with mean $1 / \mu_{\mathrm{p}}$ and that of the SU follows 


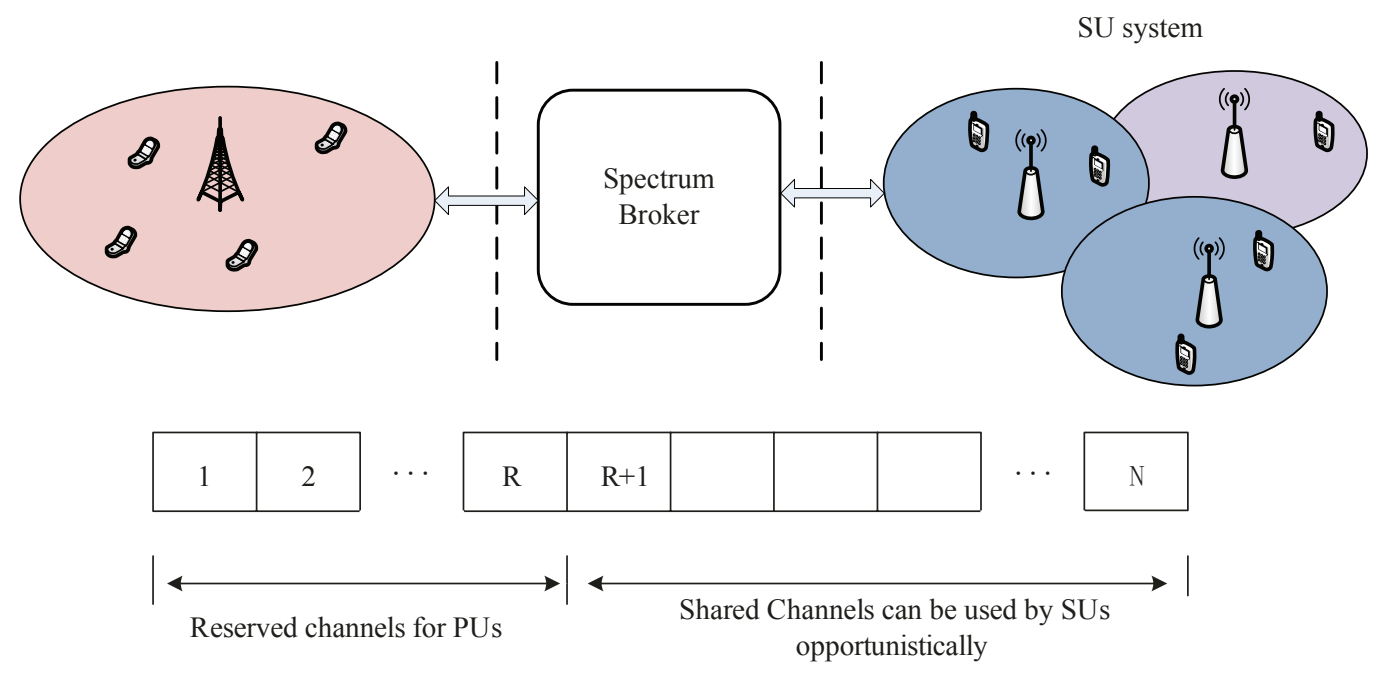

Figure 1 System model.

exponential distribution with mean $1 / \mu_{\mathrm{s}}$. As the number of spectrum holes varies with PUs traffic dramatically, we assume the traffic of SUs has much shorter average service time compared to the traffic of PUs. A first in first out buffer of size $Q$ is allocated for the secondary system.

In this section, we describe the process of spectrum sharing in the CRN as a multi-dimensional Markov chain with three state variables. The states in the model are denoted as $\left\{N_{\mathrm{p}}(t), N_{\mathrm{s}}^{\prime}(t), N_{\mathrm{s}}(t)\right\}$.

$$
P_{i, j, k}=\lim _{t \rightarrow \infty} P\left\{N_{\mathrm{p}}(t)=i, N_{\mathrm{s}}^{\prime}(t)=j, N_{\mathrm{s}}(t)=k\right\} \quad \text { repre- }
$$
sents the steady probability of state, in which $N_{\mathrm{p}}(t)=i$ is the number of PUs in the system, $N_{\mathrm{s}}^{\prime}(t)=j$ is the number of SUs in the system, $N_{\mathrm{s}}(t)=k$ is the number of SUs in service. Here, we use $(i, j, k)$ as the notation of a state in the model.

\subsection{Preemptive mechanism}

In the preemptive mechanism, a SU has to switch to another spectrum hole or stop its transmission (be preempted) as soon as a PU reclaims the channel, since PUs are given priorities over SUs. The preempted SU that ceases ongoing packet transmission will put the failed transmission packet into the buffer and wait for transmission again. However, if the buffer is full, then the SU's failed transmission packet will be dropped. The number of channels that SUs can use is a random variable, which depends on the PUs' service probability distributions. Since the number of the spectrum holes depends on the PUs' traffic, the number of SUs in service also varies with PUs' traffic. Figure 2 shows an example of the state transition diagram with $N=3, R=$ 1 . The state space of the preemptive mechanism $\Omega^{\text {pre }}$ is presented as

$$
\Omega^{\mathrm{pre}}=\left\{\begin{array}{l}
(i, j, k): 0 \leq i \leq N ; 0 \leq k \leq \min (N-R, N-i) ; \\
j=k, \text { if } 0 \leq k<\min (N-R, N-i) ; \\
k \leq j \leq k+Q, \text { if } k=\min (N-R, N-i)
\end{array}\right\} .
$$

In Figure 2, we can see that unidirectional transitions exist in the Markov chain, so that the Markov chain cannot be reversible, which means that the exact closedform solutions are non-trivial to obtain. Decomposition technique [9] is used as a tool to derive the approximate closed-form solutions of steady-state probabilities in the Markov chain. The Markov chain can be broken down into a hierarchy of groups of aggregate states. Each group of states comprises of all the states with a fixed number of PUs. Figure 2 shows that there are four groups of aggregate states and each group is circled by a line separately. All transitions between the groups are in terms of $\lambda_{\mathrm{p}}$ and $\mu_{\mathrm{p}}$. For the duration of a specific number of PUs, the states of SUs achieve equilibrium. All the transitions within a group are in terms of $\lambda_{\mathrm{s}}$ and $\mu_{\mathrm{s}}$, and the steady-state probabilities $P_{i, j, k}^{\text {pre }}$ in the preemptive mechanism can be approximated by ignoring the transitions between groups.

PUs have preemptive priorities over SUs, which implies that the equilibrium distribution of PUs can simply be modeled as a $M / M / N / N$ queueing system. $P_{i}$ represents the probability of $i$ PUs in the system, which can be derived by Erlang-B formula [9]:

$$
P_{i}=\frac{\rho_{\mathrm{p}}^{i} / i !}{\sum_{j=0}^{N} \rho_{\mathrm{p}}^{j} / j !}, \quad \text { where } \quad \rho_{\mathrm{p}}=\frac{\lambda_{\mathrm{p}}}{\mu_{\mathrm{p}}} .
$$




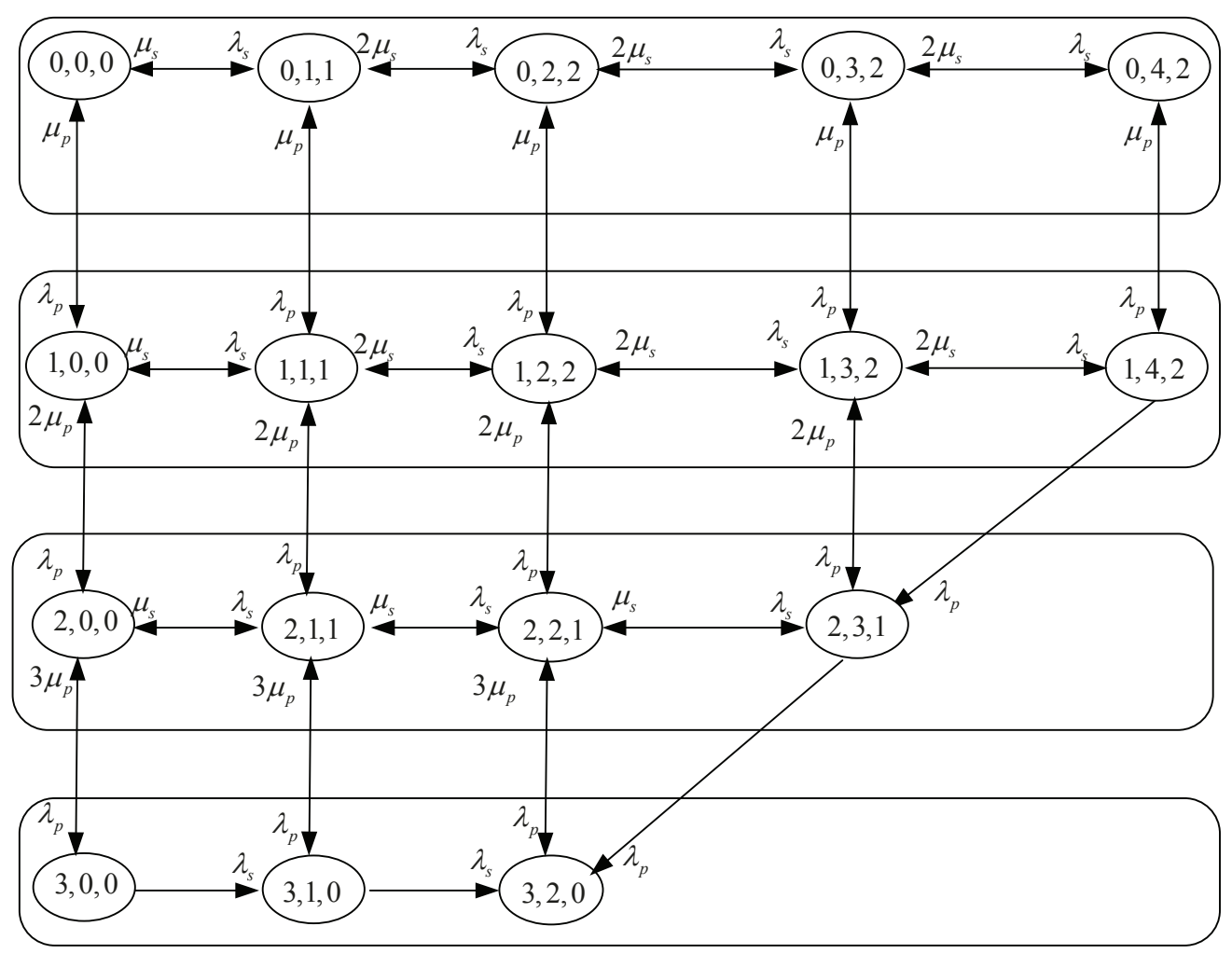

Figure 2 An example of the preemptive mechanism.

$\forall_{i} \in\{0,1, \ldots, R\}$, the $M / M / N-R / N-R+Q$ queueing system can be used to obtain $P_{i, j, \min (j, N-R)}^{\text {pre }}$, which represents the probability of $j$ SUs in the system. $\rho_{\mathrm{s}}=\lambda_{\mathrm{s}} / \mu_{\mathrm{s}}$ refers to the SU traffic load in Erlang. For simplicity, we denote $N-R=D, N-R+Q=E$.

$$
\begin{gathered}
P_{i, j, \min (j, D)}^{\mathrm{pre}}=\left\{\begin{array}{c}
P_{i} \cdot P_{i, 0}^{\mathrm{pre}} \rho_{\mathrm{s}}^{j} / j ! 0 \leq j<D \\
P_{i} \cdot \frac{P_{i, 0}^{\mathrm{pr}} \rho_{\mathrm{s}}^{j}}{D ! D^{j-D}} D \leq j \leq E
\end{array}\right. \\
P_{i, 0}^{\mathrm{pre}}=\left(\frac{\rho_{\mathrm{s}}^{D}\left(1-\left(\rho_{\mathrm{s}} / D\right)^{(Q+1)}\right)}{\left(1-\frac{\rho_{\mathrm{s}}}{D}\right) D !}+\sum_{x=0}^{D-1} \rho_{\mathrm{s}}^{x} / x !\right)^{-1}
\end{gathered}
$$

$\forall_{i} \in\{R+1, \ldots, N-1\}, P_{i, j, \min (j, N-i)}^{\text {pre }}$ can be derived from the $M / M / N-i / N-i+Q$ queueing system similarly as (2) and (3).

For $i=N$, we construct the balance equations of the states in the group. The steady-state probabilities can be easily obtained.

$$
P_{N, j, 0}^{\mathrm{pre}}=\lambda_{\mathrm{s}}^{j} P_{N, 0,0}^{\mathrm{pre}}
$$

$$
\sum_{j=0}^{Q} P_{N, j, 0}^{\mathrm{pre}}=\left(1+\lambda_{\mathrm{s}}+\cdots+\lambda_{\mathrm{s}}^{Q}\right) P_{N, 0,0}^{\mathrm{pre}}=P_{N}
$$

All the steady-state probabilities in the preemptive mechanism are given approximately in above formulas. The complete algorithm for the steady-state probabilities in the preemptive mechanism is described in Appendix A

\subsection{NP mechanism}

In the NP mechanism, PUs have no preemptive priorities over SUs. When there is no spectrum hole to switch, a SU would not vacate the channel reclaimed by a PU until the SU finishes its ongoing transmission. It means that SUs would not be forcibly terminated by PUs. Both the primary and the secondary systems can communicate with the spectrum broker through auxiliary control channels [7]. We describe the explicit interactions between the primary and the secondary systems as follows.

In the secondary system, SUs can monitor the realtime situation of the shared channels by periodic spectrum sensing. Once there is no spectrum hole, the secondary system will inform a waiting signaling to the primary system through the spectrum broker. After 
receiving this signaling, the PU who is ready to transmit will wait for a period of time and inform the secondary system the target channel that it reclaims. The SU in the specific channel will vacate the channel immediately after it finishes the ongoing transmission. If the channel can be released before the PU's waiting time is due, then the PU can access the target channel and the PU's service is only deferred. Otherwise, the PU will be blocked. Once the SUs sense that there appears a spectrum hole (a SU or PU in service left), the waiting signaling is canceled for PUs in the primary system via the spectrum broker. In the situation without waiting signaling, the proposed mechanism works in the same way as the preemptive mechanism.

In this article, we assume that the waiting time of a PU follows exponential distribution with mean $1 / \mu_{\mathrm{p}}$, which is the same as the PU's service time. Therefore, the total rate of a PU leaving the system only depends on $N_{\mathrm{p}}(t)$. This implies that the number of PUs in the system is independent of the SUs' traffic and the steady state probabilities of $N_{\mathrm{p}}(t)$ can also be derived by (1).
Figure 3 shows an example of the state transition diagram of NP mechanism with $N=3, \mathrm{R}=1$. The state space of NP mechanism $\Omega^{\text {nonpre }}$ is

$$
\Omega^{\mathrm{nonpre}}=\left\{\begin{array}{l}
S^{n}=\Omega^{\mathrm{pre}} \\
\left.S^{q}=\left\{\begin{array}{l}
(i, j, k): R+1 \leq i \leq N ; \\
\min (N-i, N-R)<k \leq \max (N-i, N-R) ; \\
k \leq j \leq k+Q
\end{array}\right\}\right\} .
\end{array}\right.
$$

In Figure 3, the shaded states represent the states with PUs queueing for transmission, and these states do not exist in preemptive mechanism. The set of states with PUs queueing is denoted as $S^{q}$, while the set of the other states in $\Omega^{\text {nonpre }}$ is denoted as $S^{n}$. In queueing states, $i+k>N$, only $N-K$ PUs are in service, $i-(N-K)$ PUs are queueing for transmission.

We use the decomposition technique to derive the approximate closed-form solutions of steady-state probabilities $P_{i, j, k}^{\text {nonpre }}$ in the proposed NP mechanism.

Step 1. For $i \in(0, \ldots, R)$, all states are in $S^{n}$, and the state transitions in each group can be modeled as $M / M$ /

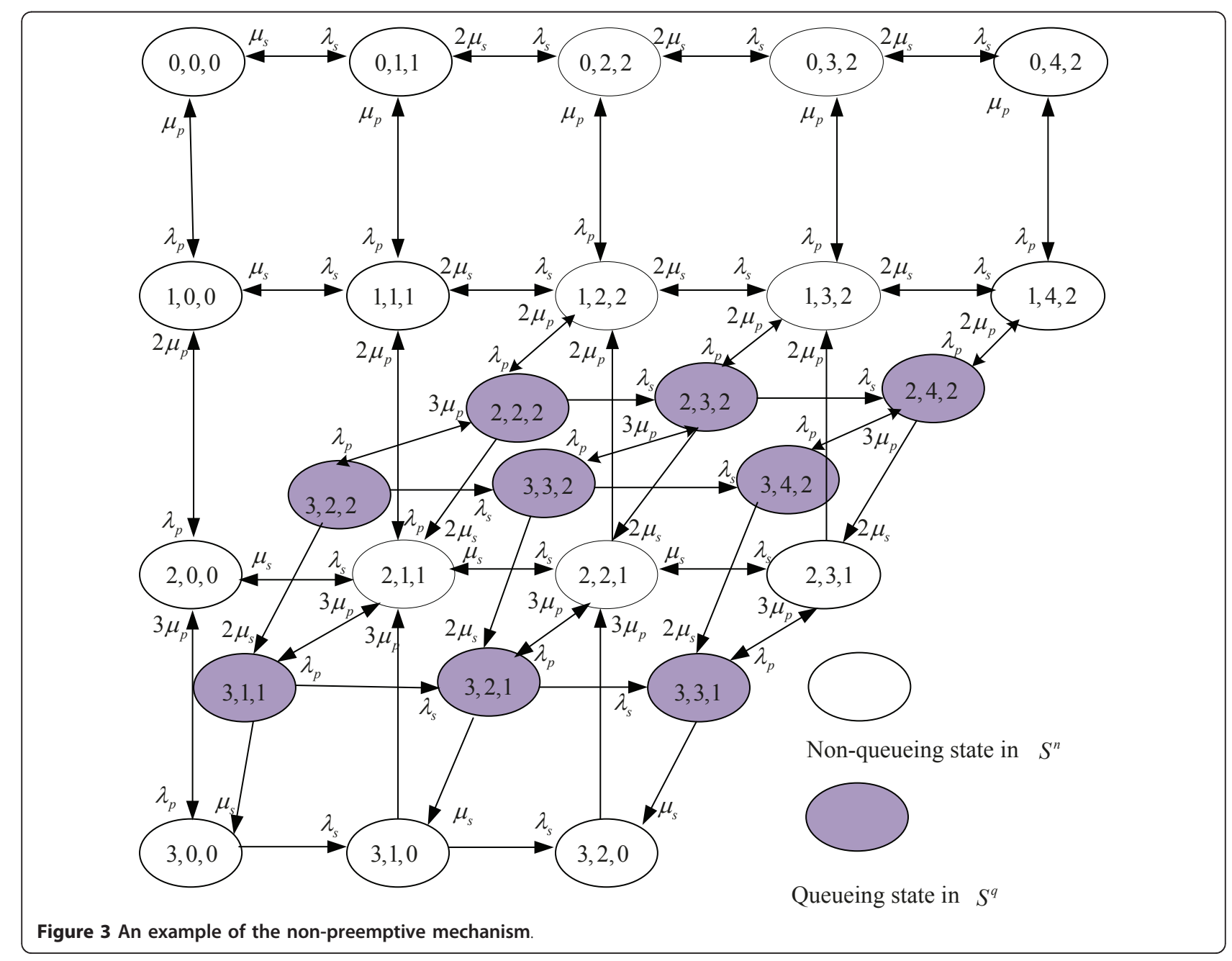


$(N-R) /(N-R)+Q$. Therefore, the steady-state probabilities of $j$ SUs in the system $P_{i, j, \min (j, N-R)}^{\text {nonpe }}$ can be derived by the same formulas as (2) and (3).

Step 2. For $i \in(R, \ldots, N-1)$, we denote the queueing states as $(i, j, k)$ to distinguish it from the non-queueing states here. The transitions into the queueing states $\{i=$ $\left.1 \leq i^{\prime} \leq N, j \leq k, k=\min (N-i, N-R)\right\}$ are only from the non-queueing states $\{i, j \leq k, k=\min (N-i, N-R)\}$, which have been obtained from last step. Figure 4 shows an example of the transition diagram between non-queueing states and queueing states.

We define the terms $F_{i, j, k}, R_{i, j, k}$ as follows.

$$
\begin{aligned}
F_{i^{\prime}, j, k} & \equiv P_{i^{\prime}, j-1, k}^{\text {nonpre }} \lambda_{s} \varphi\left(i^{\prime}, j-1, k\right) \\
& =\text { total probability flux into state }\left(i^{\prime}, j, k\right) \\
& \text { other than from }\left(i^{\prime}-1, j, k\right) \text { or }\left(i^{\prime}+1, j, k\right)
\end{aligned}
$$

in which $\phi(i, j, k)$ indicates whether the state $(i, j, k)$ exists or not, i.e. $\phi(i, j, k)=1$, if $(i, j, k) \in \Omega^{\text {nonpre }}$.

$$
\begin{aligned}
R_{i^{\prime}, j, k} & =\lambda_{s}+k \mu_{s}+\lambda_{p}+i^{\prime} \mu_{\mathrm{p}} \\
& =\text { total rate out of state }\left(i^{\prime}, j, k\right) .
\end{aligned}
$$

We use (6) and (7) to construct balance equations for the queueing states, as proposition 1 in $[10] . P_{i, j, k}^{\text {nonpre }}$ satisfies the following recursive relationship:

$$
\begin{aligned}
P_{i^{\prime}, j, k}^{\text {nonpre }} & =\Gamma_{i^{\prime}-1, j, k}+P_{i^{\prime}-1, j, k}^{\text {nonpre }} \Theta_{i^{\prime}-1, j, k .} \\
\Gamma_{i^{\prime}-1, j, k} & =\left\{\begin{array}{c}
\frac{F_{i^{\prime}, j, k}+\left(i^{\prime}+1\right) \mu_{p} \Gamma_{i^{\prime}, j, k}}{R_{i^{\prime}, j, k}-\left(i^{\prime}+1\right) \mu_{p} \Theta_{i^{\prime}, j, k}} \\
0 i^{\prime}>N
\end{array}\right.
\end{aligned}
$$

$$
\Theta_{i^{\prime}-1, j, k}=\left\{\begin{array}{c}
\frac{\lambda_{p}}{R_{i^{\prime}, j, k}-\left(i^{\prime}+1\right) \mu_{p} \Theta_{i^{\prime}, j, k}} \\
0 i^{\prime}>N
\end{array}\right.
$$

Step 3. For $i \in(R+1, \ldots, N-1)$, we can derive the nonqueueing states' equilibrium probabilities $P_{i, j, \min (j, N-i)}^{\text {nonpe }}$ according to the following balance equations. Figure 5 shows an example of the transition diagram between the queueing states with known equilibrium probabilities and the non-queueing states we are interested in.

$$
\begin{aligned}
& P_{i, 0,0}^{\text {nonpre }} \lambda_{\mathrm{s}}=P_{i, 1,1}^{\text {nonpre }} \mu_{\mathrm{s}} \\
& P_{i, 0,0}^{\text {nonpre }}+P_{i, 1,1}^{\text {nonpre }}+\cdots+P_{i, N-i+Q, N-i}^{\text {nonpre }}=P_{i}-P_{q}(i) \\
& P_{q}(i) \equiv \sum_{\forall j, k \text { s.t. }}(i, j, k) \in S^{q}
\end{aligned}
$$

The closed-form solutions of steady-state probabilities $P_{i, j, \min (j, g(i))}^{\mathrm{nonpre}}$ for the queueing states with $i \in(R+1, \ldots, N-1)$ can be written as (11). We denote $N-i=g(i), N-i+1=x(i),(N-i+1) P_{i, b, N-i+1}^{\text {nonpre }}=f_{i, b}$ here.

$$
P_{i, j, \min (j, g(i))}^{\text {nonpe }}=\left\{\begin{array}{c}
P_{i, 0}^{\text {nonpre }} \frac{\rho_{\mathrm{s}}^{j}}{j !} 1 \leq j \leq g(i) \\
P_{i, 0}^{\mathrm{nonpre}} \frac{\rho_{\mathrm{s}}^{g(i)}}{g(i) !}\left(\frac{\rho_{\mathrm{s}}}{g(i)}\right)^{j-g(i)} \\
-\sum_{a=0}^{j-x(i)}\left(\frac{\rho_{\mathrm{s}}}{g(i)}\right)^{j-x(i)-a} \sum_{b=x(i)+a} \frac{f_{i, b}}{g(i)} g(i)<j
\end{array}\right.
$$

Known Equilibrium Probabilities

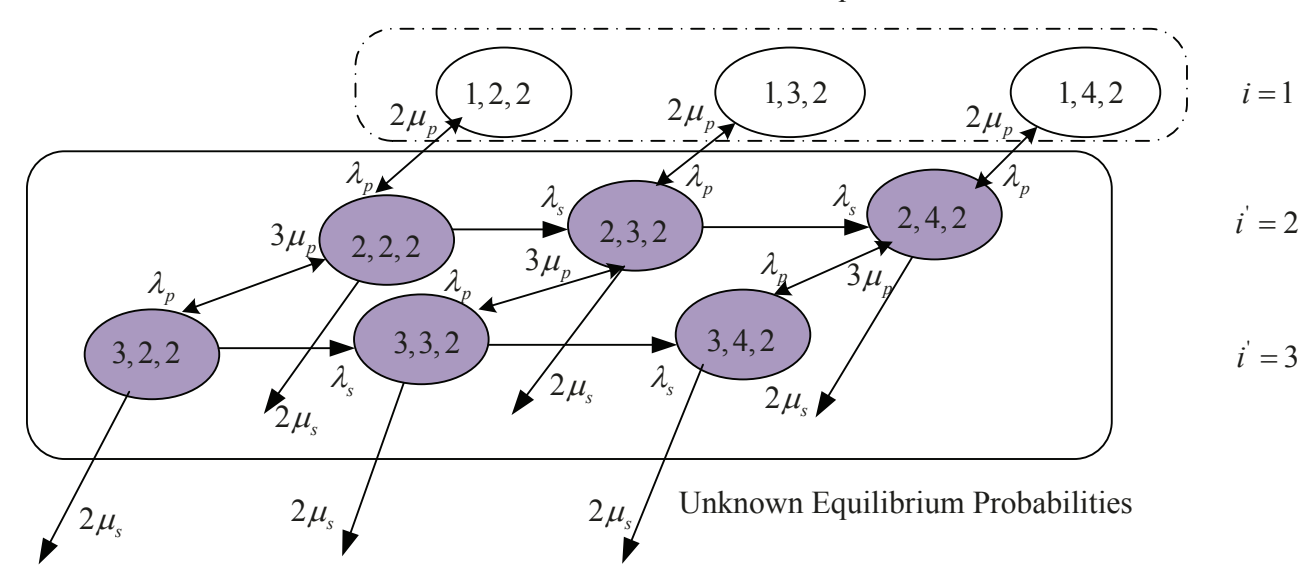

Figure 4 Decomposition solution to the queueing states with $i=R$. 


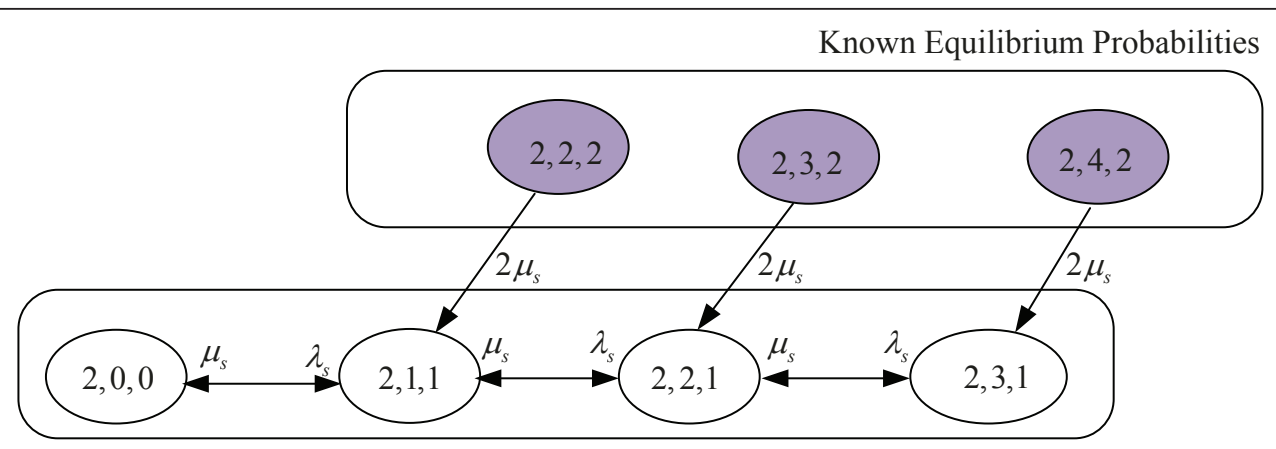

Unknown Equilibrium Probabilities

Figure 5 Decomposition solution to non-queueing states with $i=R+1$.

$$
P_{i, 0}^{\text {nonpre }}=\frac{P_{i}-P_{q}(i)+\sum_{j=x(i)}^{g(i)+Q j-x(i)}\left(\frac{\rho_{\mathrm{s}}}{g(i)}\right)^{j-x(i)-a} \sum_{b=x(i)} \frac{f_{i, b}}{g(i)}}{\sum_{j=0}^{g(i)} \frac{\rho_{\mathrm{s}}^{j}}{j !}+\frac{\rho_{\mathrm{s}}^{g(i)}}{g(i) !} \sum_{b=1}^{Q}\left(\frac{\rho_{\mathrm{s}}}{g(i)}\right)^{b}}
$$

Step 4. For $i=N$, Figure 6 shows an example of the transition diagram between states with known equilibrium probabilities and states that we are interested in.

According to the decomposition technique, local balance equation can be presented as (13). As a result, the equilibrium probabilities can easily be written as (14) and (15).

$$
\begin{aligned}
& P_{N, 1,1}^{\text {nonpre }} \mu_{\mathrm{s}}+P_{N-1,0,0}^{\text {nonpre }} \lambda_{\mathrm{p}}=P_{N, 0,0}^{\text {nonpre }}\left(\lambda_{\mathrm{s}}+N \mu_{\mathrm{p}}\right) \\
& P_{N, 0,0}^{\text {nonpre }}=\frac{P_{N, 1,1}^{\text {nonpre }} \mu_{\mathrm{s}}+P_{N-1,0,0}^{\text {nonpre }} \lambda_{\mathrm{p}}}{\lambda_{\mathrm{s}}+N \mu_{\mathrm{p}}}
\end{aligned}
$$

$$
P_{N, j, 0}^{\text {nonpre }}=\frac{P_{N, j+1,1}^{\text {nonpre }} \mu_{\mathrm{s}}+P_{N, j-1,0}^{\text {nonpre }} \lambda_{\mathrm{s}}}{\lambda_{\mathrm{s}}+N \mu_{\mathrm{p}}} 1 \leq j \leq Q
$$

All the steady-state probabilities in the NP mechanism are given approximately by above four steps. The complete algorithm for calculating the steady-state probabilities in the NP mechanism is presented in Appendix B. The main purpose of deriving the steady-state probabilities is to evaluate the performance metrics in the joint leasing and sensing-based CRN.

\section{Performance metrics}

QoS is defined as the ability of the network to provide a service at an assured service level, which is also the performance evaluation standard of the network. A user perceives the QoS in the specific network in terms of, for example, usability, retainability, and integrity of the service [13]. Blocking probability is the probability that a

\section{Known Equilibrium Probabilities}

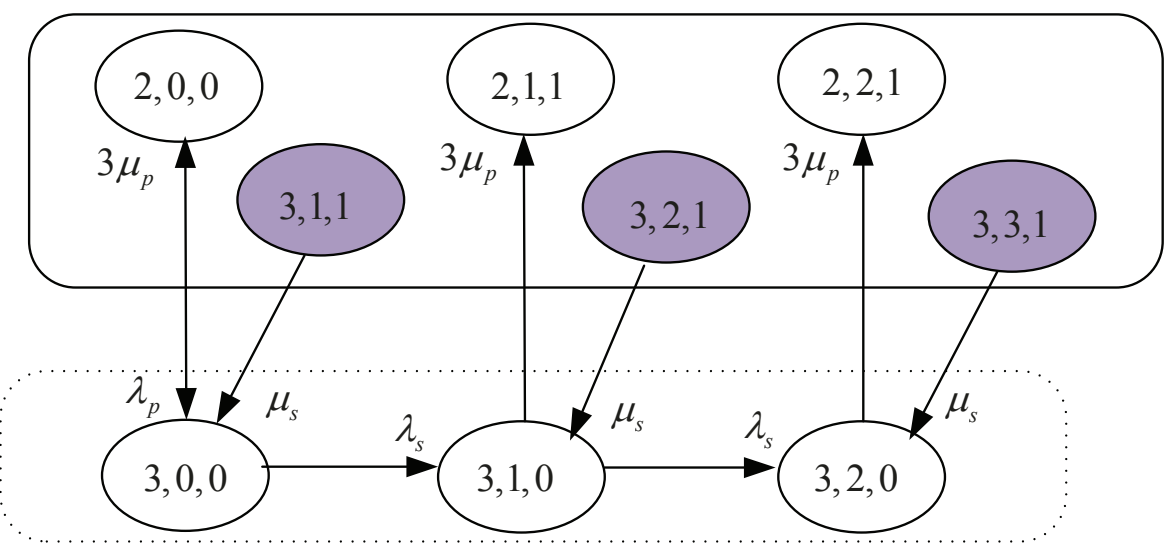

Unknown Equilibrium Probabilities

Figure 6 Decomposition solution to non-queueing states with $i=N$. 
user is blocked when it is trying to access the system, which reflects the usability of the network. Force-termination probability is the probability that a user has to stop its ongoing transmission. The force-termination probability can reflect the retainability of the service. As the service integrity relates to the delay of data transmission, mean system delay and mean waiting time are also in our considerations.

For evaluating the spectrum-sharing mechanisms in the CRN, metrics that we consider include force-termination probability of SU $P_{\mathrm{FT}-\text { su }}$, mean system delay of SU $T_{\text {Delay-su }}$, mean waiting time of PU $t_{\text {wait-pu }}$, and blocking probability of PU $P_{\mathrm{BL}-\mathrm{pu}}$. The expressions of these metrics are described as follows. We define $f(i) \equiv$ $\min (N-i, N-R)$.

\subsection{Metrics in the preemptive mechanism}

The force-termination probability and dropping probability of SU are obtained as

$$
\begin{gathered}
P_{\mathrm{FT} \text { - su }}^{\mathrm{pre}}=\frac{\sum_{i=R}^{N-1} \sum_{q=0}^{Q} \lambda_{p} P_{i, N-i+q, N-i}^{\mathrm{pre}}}{\lambda_{\mathrm{s}}\left(1-P_{\mathrm{BL}-\mathrm{su}}^{\mathrm{pre}}\right)}, \\
P_{\text {Drop - su }}^{\mathrm{pre}}=\frac{\sum_{i=R}^{N-1} \lambda_{p} P_{i, N-i+Q, N-i}^{\mathrm{pre}}}{\lambda_{\mathrm{s}}\left(1-P_{\mathrm{BL} \text { - su }}^{\mathrm{pre}}\right)},
\end{gathered}
$$

in which $P_{\mathrm{BL}-\mathrm{su}}^{\mathrm{pre}}=\sum_{i=0}^{N} P_{i, f(i)+\mathrm{Q}, f(i)}^{\mathrm{pre}}$

The force-termination probability of SU $P_{\mathrm{FT}-\text { su }}$ represents the probability that the SU in service has to stop transmission because of the channel reclaimed by a PU.

The mean system delay of SU $T_{\text {Delay - su }}^{\text {pre }}$ contains the SU's transmission time and waiting time in the buffer. It can be written as

$$
T_{\text {Delay - su }}^{\text {pre }}=\frac{1}{\left(1-P_{\mathrm{BL} \text { - su }}^{\text {pre }}\right)\left(1-P_{\text {Drop - su }}^{\text {pre }}\right)} \sum_{i=1}^{N} t_{i}^{\text {pre }} P_{i} .
$$

when $0 \leq i \leq \mathrm{N}-1, t_{i}^{\text {pre }}$ represents the system delay, given that $i$ PUs are in the system and spectrum holes exist. There are two different situations here. In one situation, the SU has occupied a spectrum hole, and the system delay correspondingly equals to the mean service time of $\mathrm{SU} 1 / \mu_{\mathrm{s}}$. In the other situation, the $\mathrm{SU}$ is in the buffer with $q$ SUs waiting ahead, and the system delay is denoted as

$$
t_{i, f(i)+q, f(i)}^{\mathrm{pre}}=\left(\frac{1}{\mu_{\mathrm{s}}}+\frac{q+1}{f(i) \mu_{\mathrm{s}}}\right) .
$$

$$
t_{i}^{\mathrm{pre}}=\sum_{k=0}^{f(i)-1} P_{i, k, k}^{\mathrm{pre}}\left(\frac{1}{\mu_{\mathrm{s}}}\right)+\sum_{q=0}^{\mathrm{Q}-1} P_{i, f(i)+q, f(i)}^{\mathrm{pre}} t_{i, f(i)+q, f(i)}^{\mathrm{pre}}
$$

when $i=N$, no spectrum hole exists. The $\mathrm{SU}$ has to wait for the appearance of a spectrum hole and a queueing time of $j$ SUs which are in front of it in the buffer. $t_{i}^{\text {pre }}=\sum_{j=0}^{Q-1} P_{i, j, 0}\left(\frac{1}{N \mu_{\mathrm{p}}}+\frac{(j+1)}{\mu_{\mathrm{s}}}\right)$.

The blocking probability of $\mathrm{PU}$ is obtained as $P_{\mathrm{BL}-\mathrm{pu}}^{\mathrm{pre}}=P_{N}$. The mean waiting time of PU $t_{\text {wait }-\mathrm{pu}}^{\text {pre }}=0$, since PUs in the preemptive mechanism have priorities over SUs.

\subsection{Metrics in the NP mechanism}

The mean system delay of SU $T_{\text {Delay - su }}^{\text {nonpre }}$ can be presented as

$$
T_{\text {Delay - su }}^{\text {nonpre }}=\frac{1}{\left(1-P_{\mathrm{BL}-\mathrm{su}}^{\mathrm{nonpre}}\right)} \sum_{i=1}^{N}\left(t_{i-\text { nonque }}^{\text {nonpe }}+t_{i-\text { que }}^{\text {nonpre }}\right) P_{i}
$$

The blocking probability of SU in the NP mechanism is $P_{\mathrm{BL} \text { - su }}^{\text {nonpre }}=\sum_{i=0}^{R} P_{i, N-R+Q, N-R}^{\text {nonpre }}+\sum_{i=R+1}^{N} \sum_{k=N-i}^{N-R} P_{i, k+Q, k}^{\text {nonpre }} . t_{i-\text { nonque }}^{\text {nonpre }}$ and $t_{i \text {-que }}^{\text {nonpre }}$ represent the system delay of the states without and with PUs queueing, respectively, given that $i$ PUs are in the system. The analysis process is the same as the derivation of $T_{\text {Delay - su }}^{\text {pre }}$ in the last subsection. Due to the limited length of this article, the detail of analysis is omitted.

When $0 \leq i \leq N-1$, then

$$
\begin{aligned}
& t_{i_{-} \text {nonque }}^{\text {nonpre }}=\sum_{k=0}^{f(i)-1} P_{i, k, k}^{\text {nonpre }}\left(\frac{1}{\mu_{\mathrm{s}}}\right)+\sum_{q=0}^{Q-1} P_{i, f(i)+q, f(i)}^{\text {nonpre }} t_{i, f(i)+q, f(i)^{\prime}}^{\text {nonpre }} \\
& t_{i, f(i)+q, f(i)}^{\text {nonpre }}=\left(\frac{1}{\mu_{\mathrm{s}}}+\frac{q+1}{f(i) \mu_{\mathrm{s}}}\right) .
\end{aligned}
$$

When $i=N$, then

$$
t_{i \text { nonpre }}^{\text {none }}=\sum_{j=1}^{\mathrm{Q}-1} P_{i, j, 0}^{\text {nonpre }}\left(\frac{1}{N \mu_{\mathrm{p}}}+\frac{(j+1)}{\mu_{\mathrm{s}}}\right) .
$$

$t_{i-q u e}^{\text {nonpre }}$ satisfies the following recursive relationship:

$$
t_{i-\text { que }}^{\text {nonpre }}(i, j, k)=\sum_{k=N-i+1}^{N-R} \sum_{j=k}^{k+Q-1} P_{i, j, k}^{\text {nonpre }}\left(\frac{1}{k \mu_{\mathrm{s}}}+t_{i-\text { que }}^{\text {nonpe }}(i, j-1, k-1)\right),
$$

in which $R+1 \leq i \leq N$. When $k-1=N-i$, then

$$
t_{i-\text { que }}^{\text {nonpre }}(i, j-1, k-1)=P_{i, N-i+q, N-i}^{\text {nonpre }} t_{i, N-i+q, N-i}^{\text {nonpre }} \text {. }
$$


The blocking probability of $\mathrm{PU}$ is obtained as $P_{\mathrm{BL}-\mathrm{pu}}^{\text {nonpre }}=P_{N}+P_{\mathrm{BL}-\text { extra }} \cdot P_{\mathrm{BL} \text { - extra }}$ refers to the extra blocking probability caused by the waiting requirement raised by SUs.

$$
P_{\mathrm{BL} \text { - extra }}=\sum_{i=R+1}^{N} \sum_{j=k}^{k+Q} \sum_{k=\min (N-i, N-R)}^{\max (N-i, N-R)} P_{i, j, k}^{\text {nonpre }} \cdot \frac{i-(N-k)}{i}
$$

The mean waiting time of PU $t_{\text {wait - pu }}^{\text {nonpre }}$ is given by

$$
t_{\text {wait - pu }}^{\text {nonpre }}=\mathrm{AQ}_{\mathrm{pu}} /\left(\lambda_{\mathrm{p}}\left(1-P_{\mathrm{BL}-\mathrm{pu}}^{\mathrm{nonpre}}\right)\right) .
$$

The mean number of queueing $P U s Q_{p u}$ is

$$
\mathrm{AQ}_{\mathrm{pu}}=\sum_{\forall(i, j, k) \in S^{q}} \max \{0, i-(N-k)\} \cdot P_{i, j, k}^{\text {nonpre }} .
$$

The mean waiting time of PU refers to the average extra time that the PU spends on waiting due to the introduction of the NP mechanism in the CRN.

\section{Simulation results and discussion}

In the above two sections, we have derived all the approximate equilibrium probabilities and the expressions of performance metrics in two spectrum-sharing mechanisms. For performance evaluation, first we will give the numerical results to verify the feasibility of approximate solutions to the equilibrium probabilities. Then, these two spectrum-sharing mechanisms are compared and influences of the system parameters are taken into consideration. In the simulation, if not specially mentioned we assume that $N=5, R=2, Q=2, \mu_{\mathrm{p}}=1 /$ $10, \mu_{\mathrm{s}}=5, \lambda_{\mathrm{p}}=1$, in which $\left(1 / \mu_{\mathrm{p}}\right) /\left(1 / \mu_{\mathrm{s}}\right)>>1$. We evaluate the performance metrics versus $\lambda_{s}$, which ranges from 0.2 to 2 . In the following figures, AR and SR are the abbreviations for analytical results and simulation results, respectively, while $\mathrm{P}$ and NP represent the preemptive mechanism and NP mechanism, respectively. Two figures compose a group, and each group of figures exhibits the system parameters' influences on the performance metrics.

Figures 7 and 8 show the analytical results of performance metrics calculated by the approximate closedform solutions of the steady-state probabilities. To verify the feasibility of the approximation, we compare the analytical results with the exact numerical results for both the P and the NP mechanisms. The numerical results are carried out by Monte Carlo experiments. We can see that the analytical results and numerical results are hardly distinguishable. The closed-form solutions of the steady-state probabilities are well approximated and they can be used to analyze the performance metrics. For brevity, the numerical results are not exhibited in the rest of the article.
In Figure 7, the left subfigure shows that the mean system delay of SU $T_{\text {Delay-su }}$ increases with $\lambda_{\mathrm{s}}$. $T_{\text {Delay - su }}^{\text {nonpre }}$ is always smaller than $T_{\text {Delay - su }}^{\text {pre }}$ and the difference between $T_{\text {Delay - su }}^{\text {pre }}$ and $T_{\text {Delay - su grows with }}^{\text {nonpre }} \lambda_{\mathrm{s}}$ and $1 / \mu_{\mathrm{s}}$. The right subfigure shows $P_{\mathrm{FT} \text { - su }}^{\text {pre }}$ increases with both $\lambda_{\mathrm{s}}$ and $1 / \mu_{\mathrm{s}}$, while $P_{\mathrm{FT} \text {-su }}^{\text {nonpre }}$ stays at zero. From above descriptions, we can see that the NP mechanism improves the QoS of SU in the CRN.

On the other hand, Figure 8 shows the QoS loss of PU in the NP mechanism. $t_{\text {wait - pu }}^{\text {pre }}$ stays at zero, while $t_{\text {wait - pu }}^{\text {nonpre }}$ increases with $\lambda_{\mathrm{s}}$ and $1 / \mu_{\mathrm{s}}$. The NP mechanism leads to a growing blocking probability of PU in terms of $\lambda_{\mathrm{s}}$ and $1 /$ $\mu_{\mathrm{s}}$. A QoS tradeoff between the primary and the secondary systems can be achieved in the NP mechanism. It is because that a PU would not preempt a SU until the SU finishes its ongoing transmission when there is no spectrum hole to handoff. For QoS improvement of SUs, the NP mechanism turns into a better choice than the preemptive mechanism. The traffic parameters are key factors that influence the performance metrics. As $\lambda_{\mathrm{s}}$ and $1 / \mu_{\mathrm{s}}$ increase, the advantages of the NP mechanism become more prominent.

In the NP mechanism with $\lambda_{\mathrm{s}}=2, \mu_{\mathrm{s}}=5$, a PU spends the mean waiting time of $0.04 \mathrm{~s}$ (which accounts for $0.4 \%$ of the mean service time of PU) on queueing for transmission, and the PU also gains an extra blocking probability of 0.0034 (which accounts for $0.6 \%$ of the blocking probability of PU) because its waiting time is due. In return, the force-termination probability of SU decreases by $16 \%$ and the mean system delay of SU decreases by 0.06 (which accounts for $30 \%$ of the mean service time of SU). The results show that, significant improvement of SUs' QoS can be acquired with an acceptable loss of PUs' QoS.

Figures 9 and 10 show the influences of $\lambda_{p}$ and $\lambda_{s}$ on the performance metrics. The left subfigure in Figure 9 shows that $T_{\text {Delay-su }}$ increases with $\lambda_{\mathrm{p}}$ and $\lambda_{\mathrm{s}}$, and $T_{\text {Delay - su }}^{\text {pre }}$ is always larger than $T_{\text {Delay - su. }}^{\text {nonpre }}$ The differences between $T_{\text {Delay - su }}^{\text {pre }}$ and $T_{\text {Delay - su }}^{\text {nonpre }}$ change insignificantly with $\lambda_{\mathrm{p}}$. The

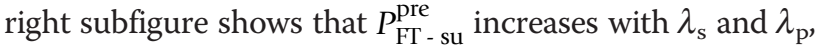
while $P_{\mathrm{FT} \text {-su }}^{\text {nonpre }}$ stays at zero. Figure 10 shows that there exists mean waiting time of PU $t_{\text {wait - pu }}^{\text {nonpre }}$ in the NP mechan-

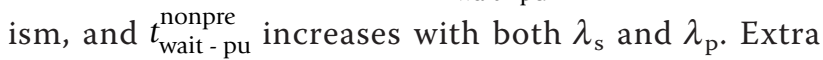
blocking probability of PU is also caused when the PU's waiting time is due in the NP mechanism. As a result, we can get the same conclusion that a QoS tradeoff is achieved between the primary and the secondary systems in the NP mechanism.

Figures 11 and 12 constitute our third simulation group. In this group, the performance metrics with different reserved channels are revealed. $R$ represents the 

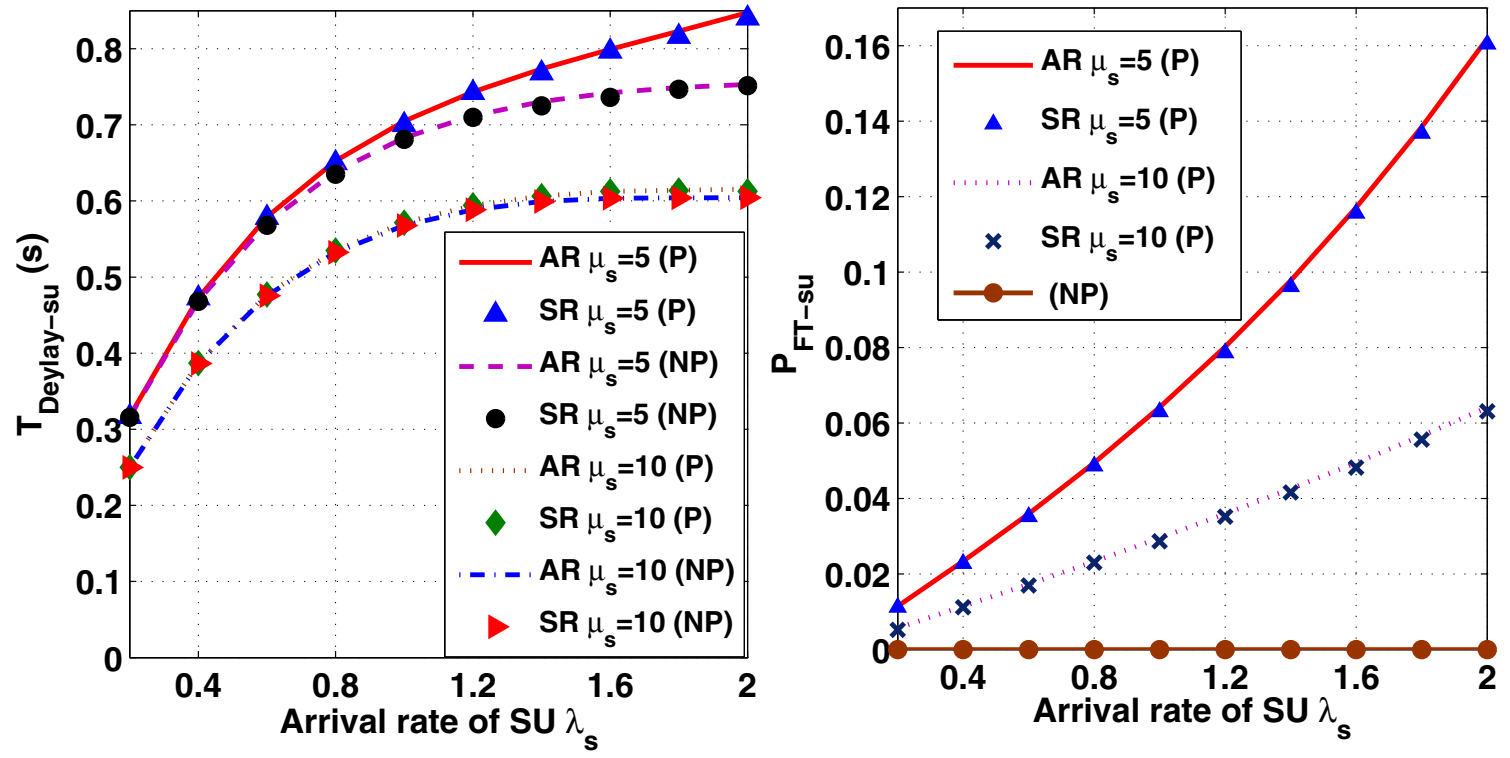

Figure 7 The mean system delay and the force-termination of SU with different mean service time of SU.

number of channels that are reserved only for PUs, $N-R$ is the number of shared channels that can be shared by PUs and SUs. Similar analysis can be done to these two figures, and the influence of system parameter $R$ on both the primary and the secondary systems can be derived easily. In addition, we also give the simulation results with other system parameters in Appendix C, such as buffer size $Q$ and total number of channels $N$. All of the simulation results show that the NP mechanism significantly improves the QoS of SUs with an acceptable QoS degradation of PUs. The performance analysis of these two spectrum-sharing mechanisms verifies that the proposed NP mechanism outperforms the preemptive mechanism in the joint leasing and sensing-based CRN.
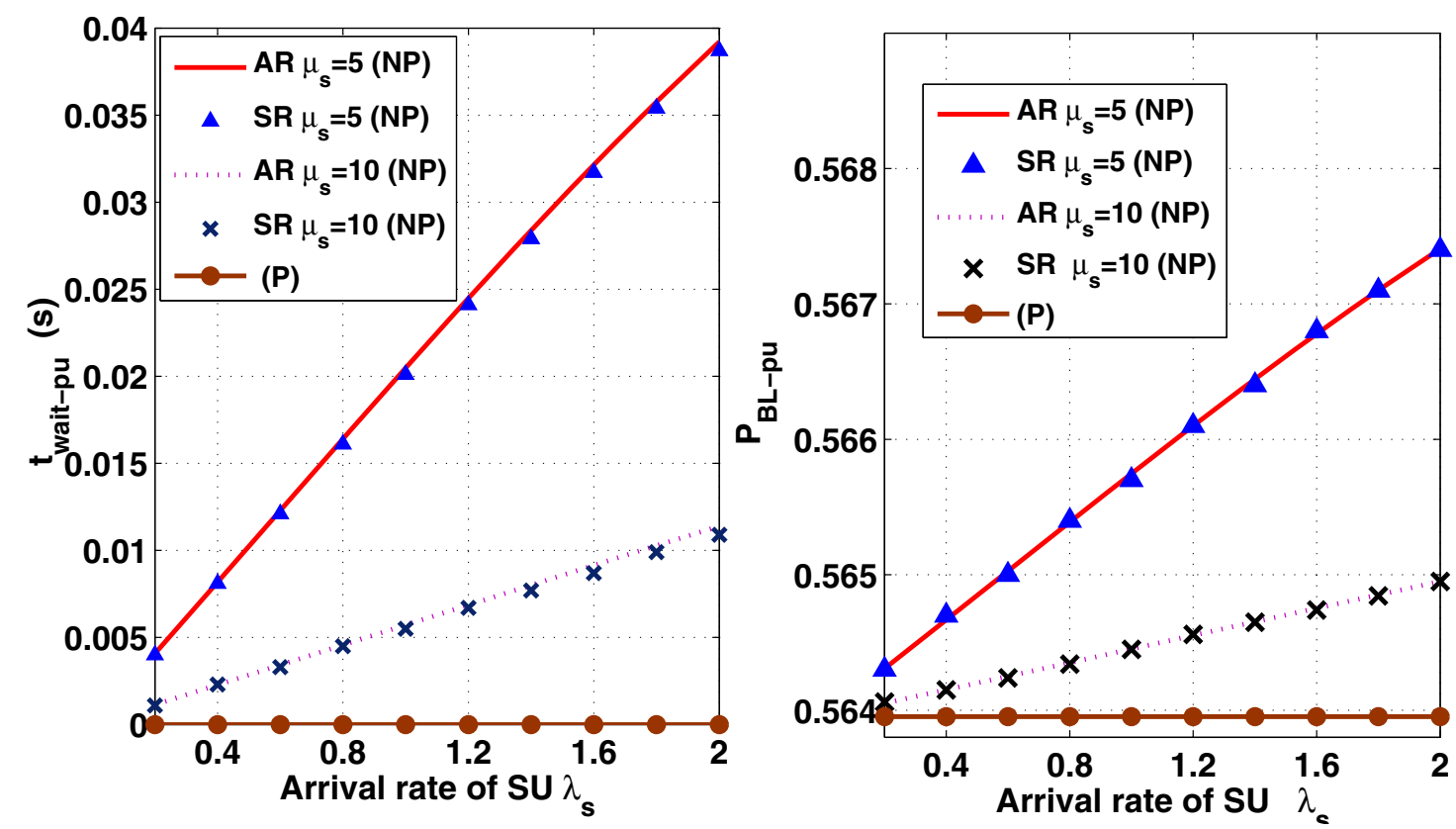

Figure 8 The mean waiting time and the blocking probability of PU with different mean service time of SU 

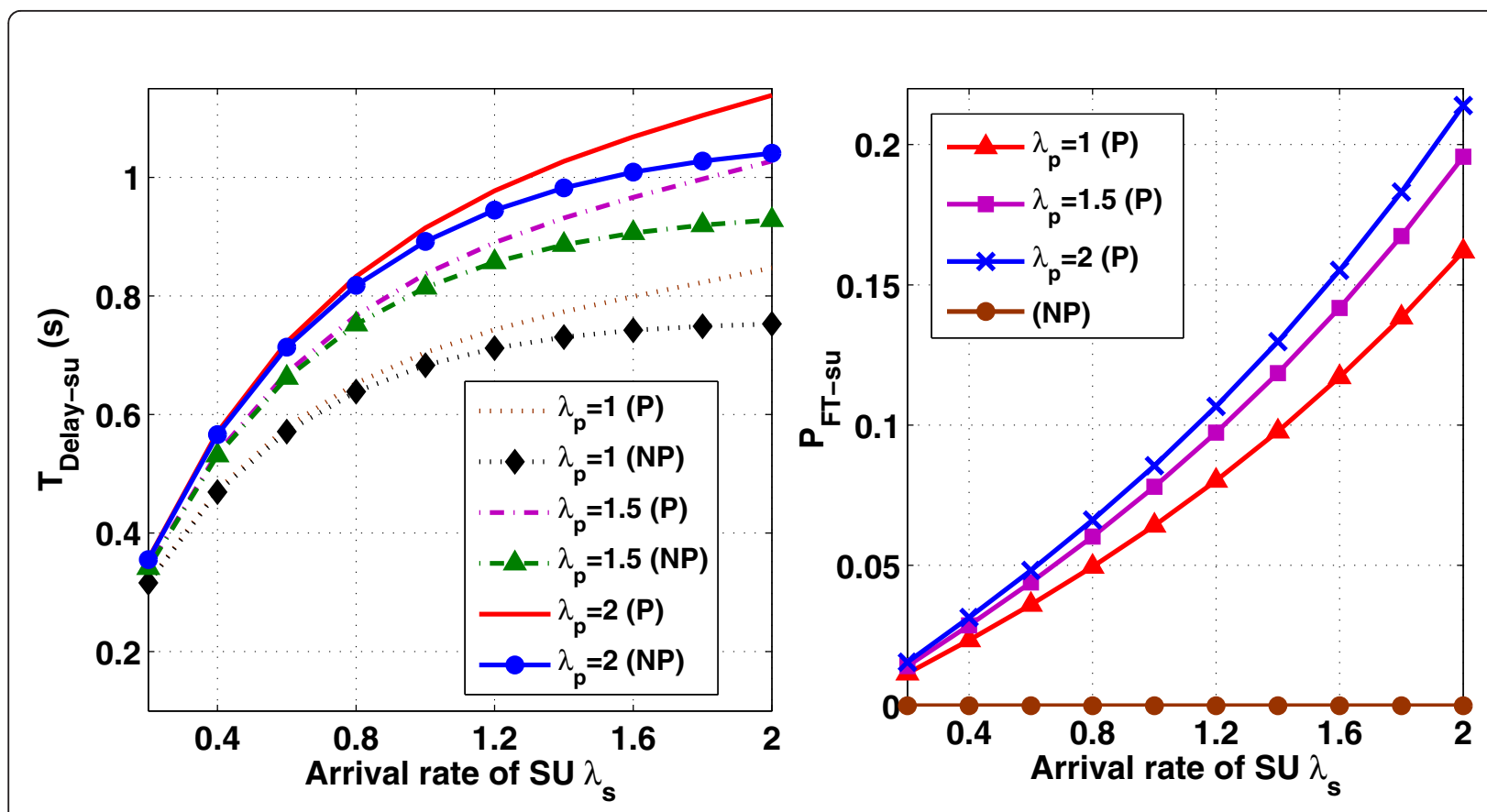

Figure 9 The mean system delay and the force-termination of SU with different arrival rates of PU

\section{Conclusion}

In the joint leasing and sensing-based CRN, the primary system leases its spectrum usage rights of shared channels to secondary system, and gets payoff from the secondary system as SUs opportunistically access the shared channels by sensing. Different from traditional sensing-based CRNs, QoS guarantee for SUs has to be considered in spectrum-sharing mechanism design. In
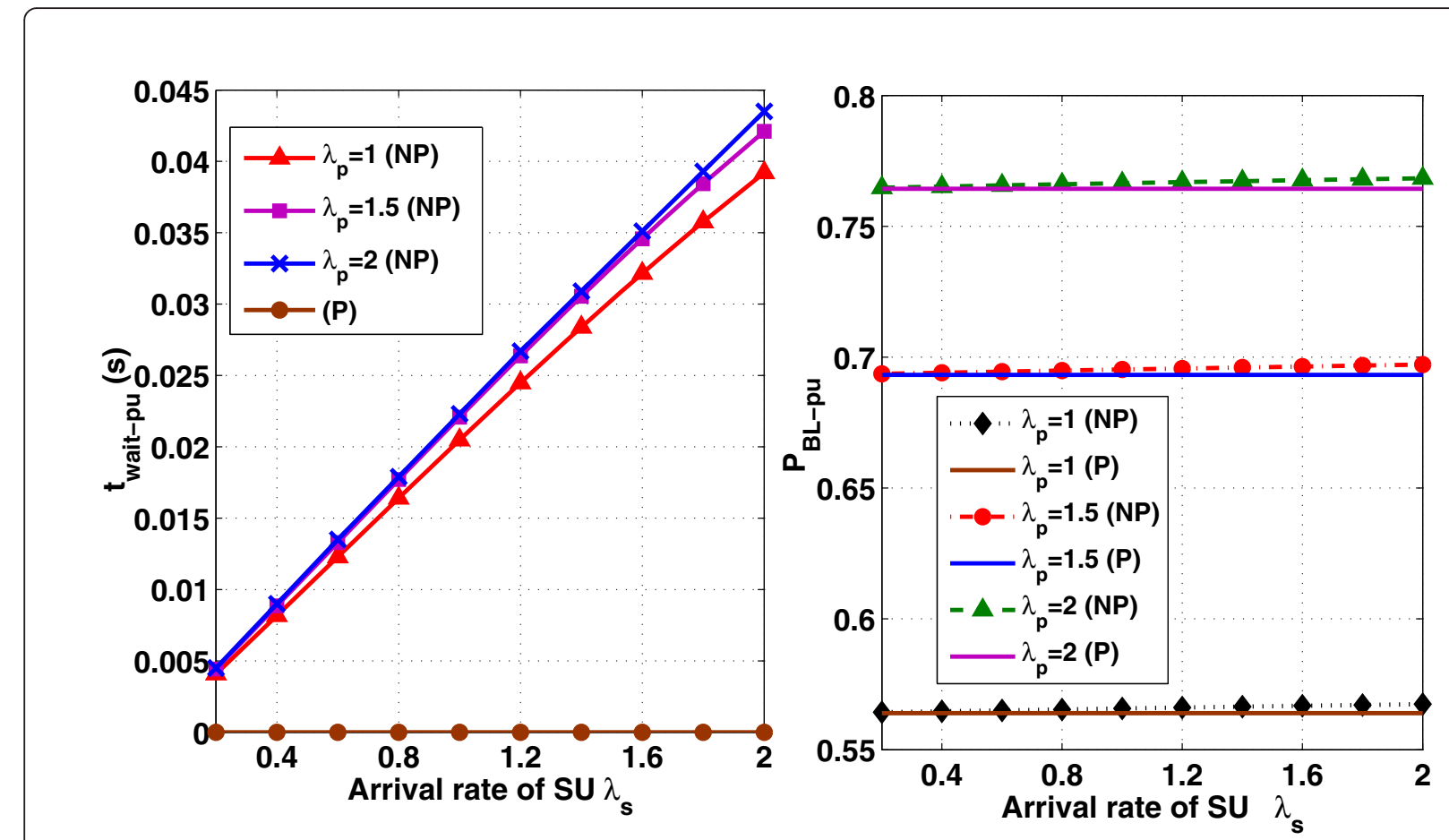

Figure 10 The mean waiting time and the blocking probability of PU with different arrival rates of PU 


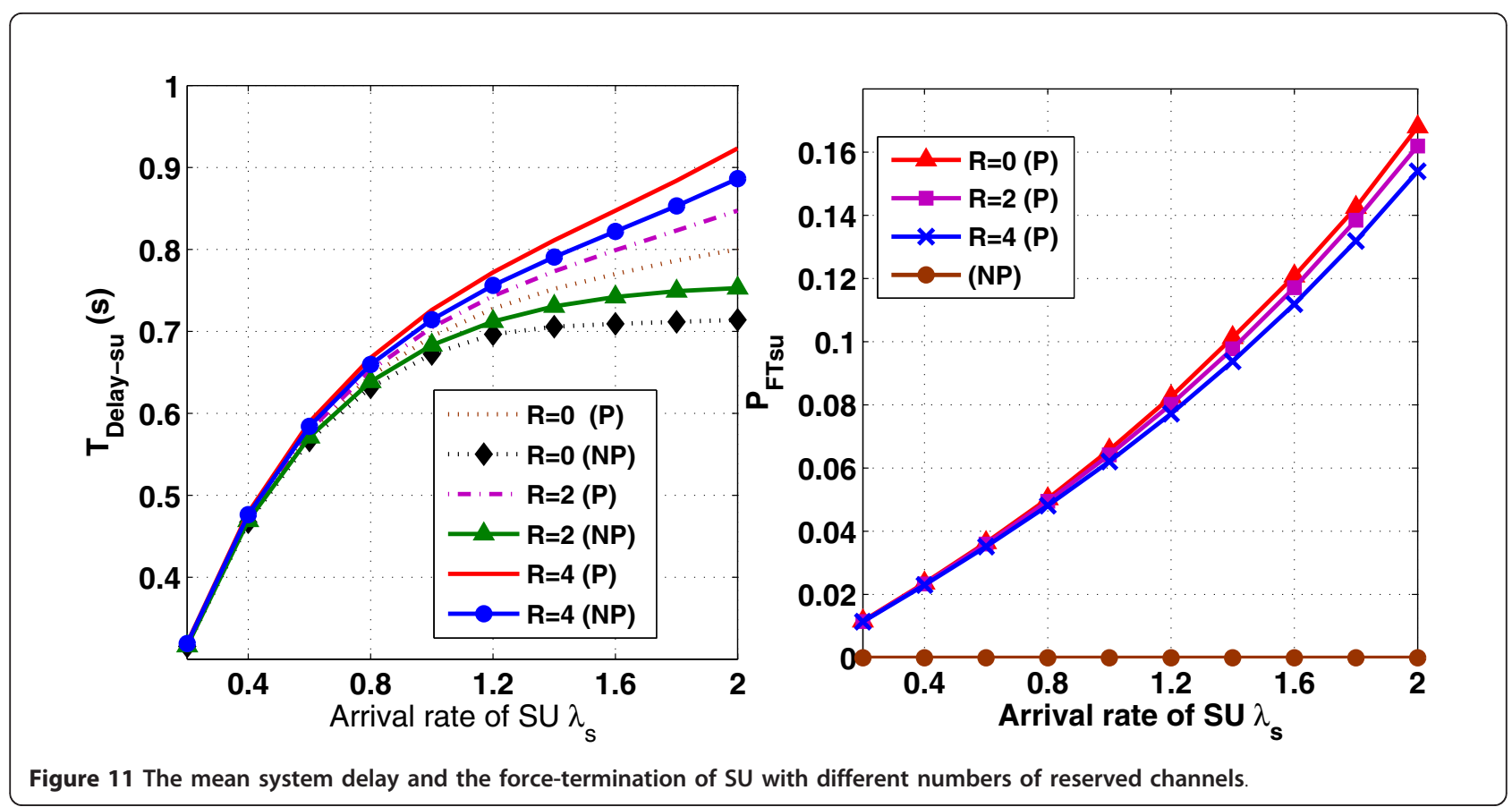

this article, we propose a NP spectrum-sharing mechanism in the joint leasing and sensing-based CRN. We have modeled both the NP mechanism and the preemptive mechanism based on multi-dimensional Markov chains. The closed-form solutions of steady-state probabilities in the two mechanisms are derived approximately by a one-dimensional decomposing method. The expressions of performance metrics including mean system delay of SU and mean waiting time of PU are also described. The approximate analytical results are verified by simulation results, which demonstrate that the closed-form solutions of the steady-state probabilities can be used to estimate the performance of the spectrum-sharing mechanisms. With the analytical solutions, the performance metrics can easily be obtained. In addition, we have discussed the impacts of system

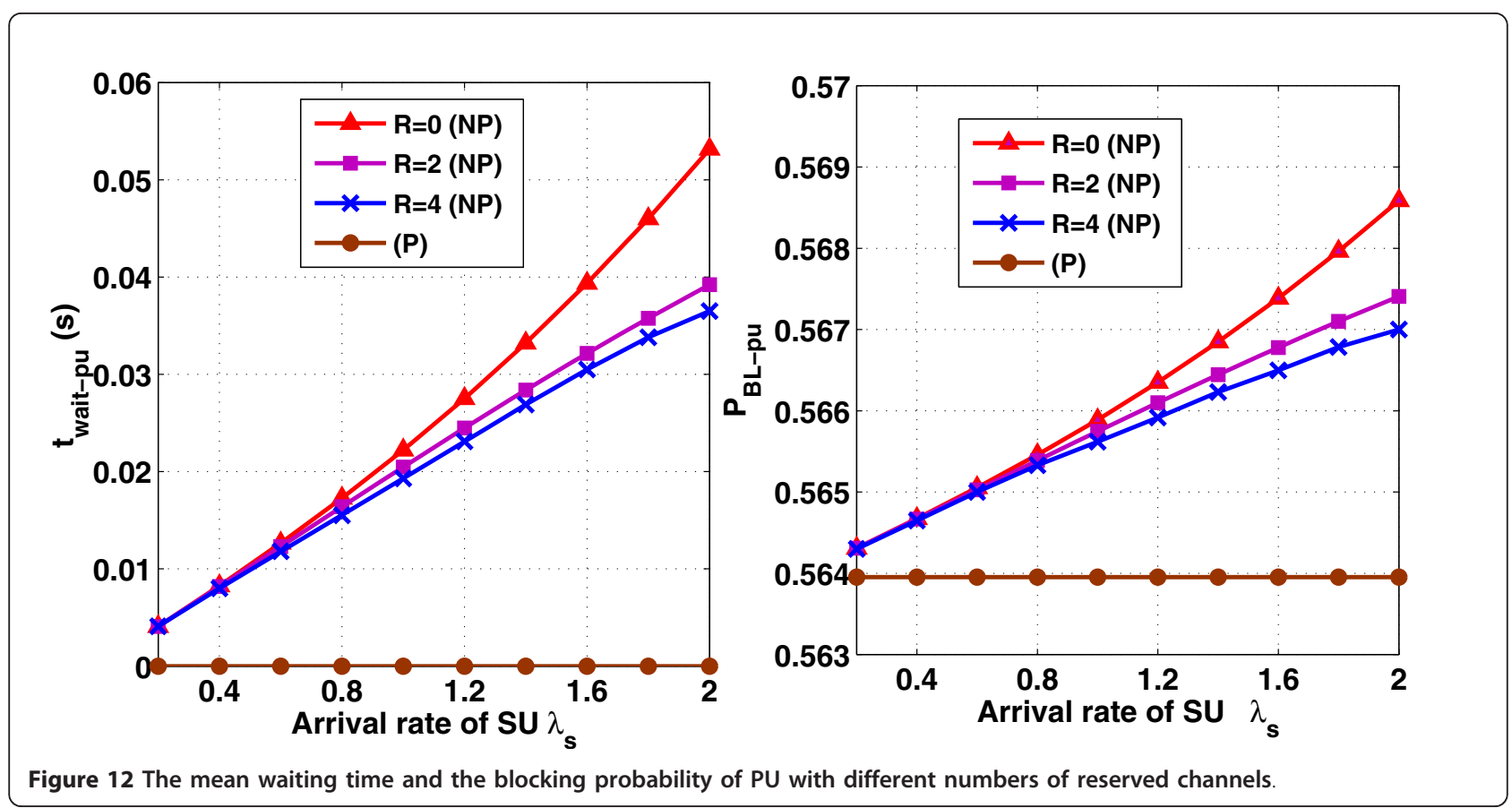




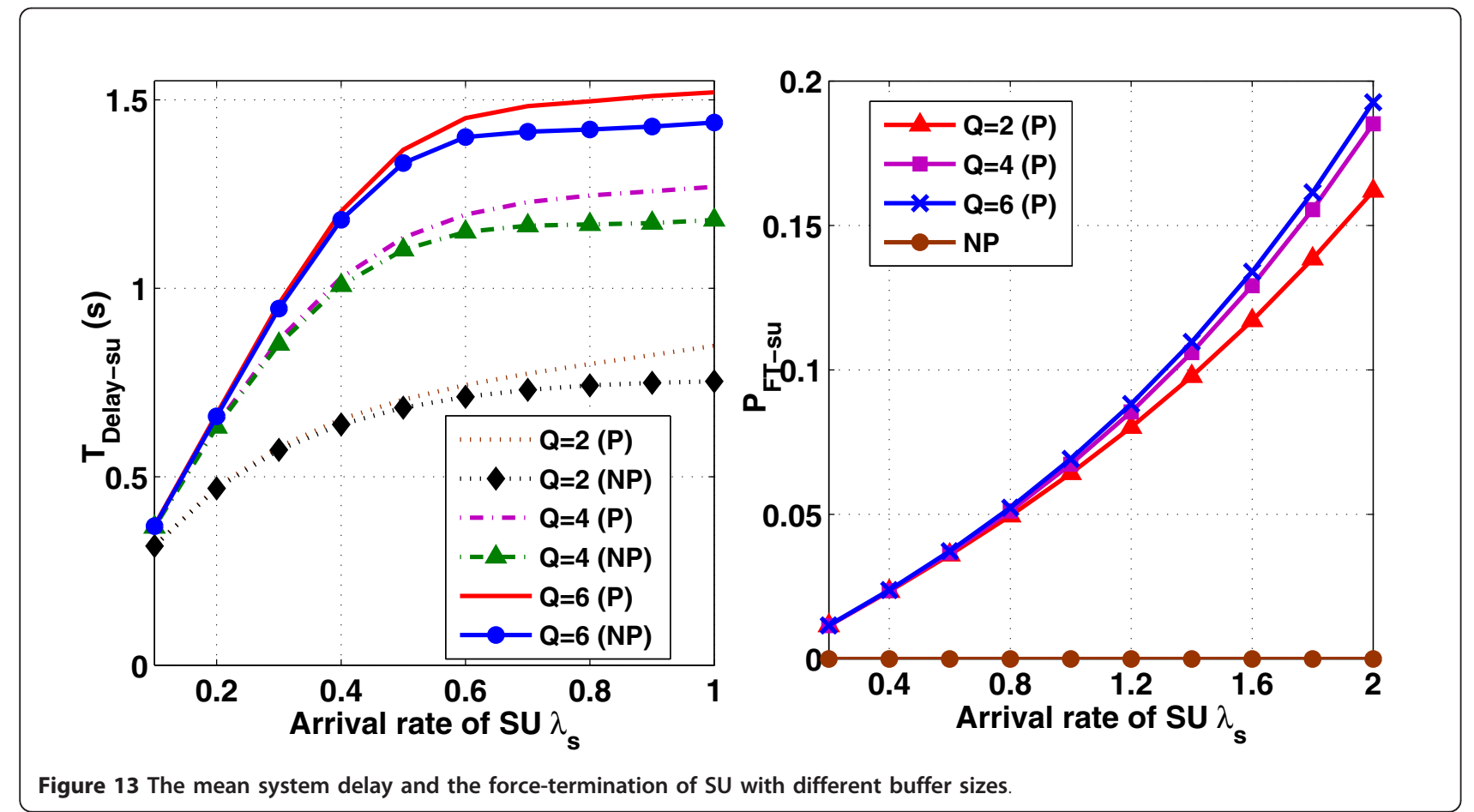

parameters such as arrival rate, service time, buffer size, and number of available channels on performance metrics. For comparison, the performance of traditional preemptive spectrum-sharing mechanism has also been analyzed and the results show that the proposed NP mechanism significantly improves the SUs' QoS with an acceptable QoS degradation of PUs. According to the performance analysis, the system parameters have impacts on the QoS tradeoff between PUs and SUs. How to balance the QoS tradeoff between PUs and SUs

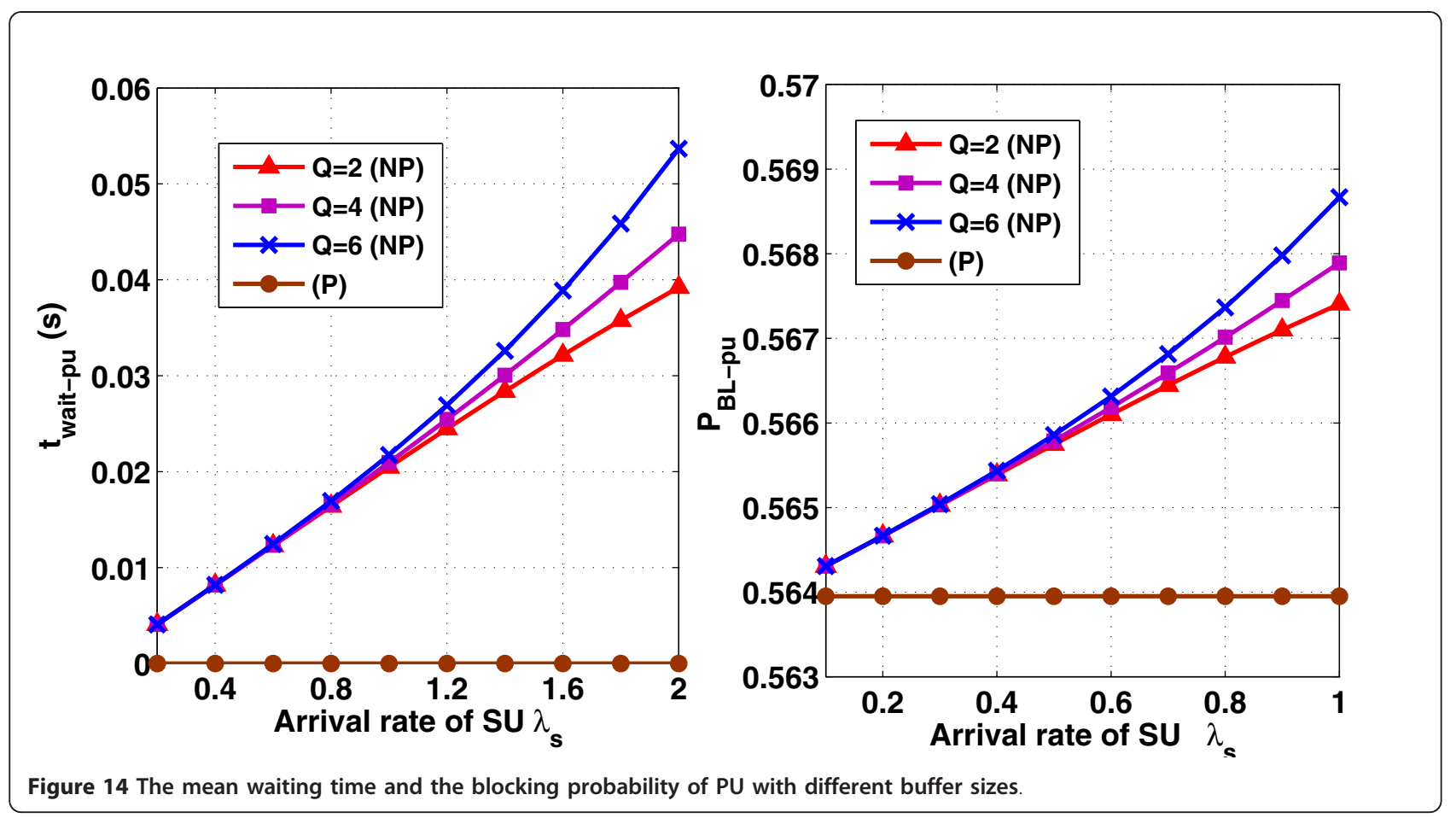



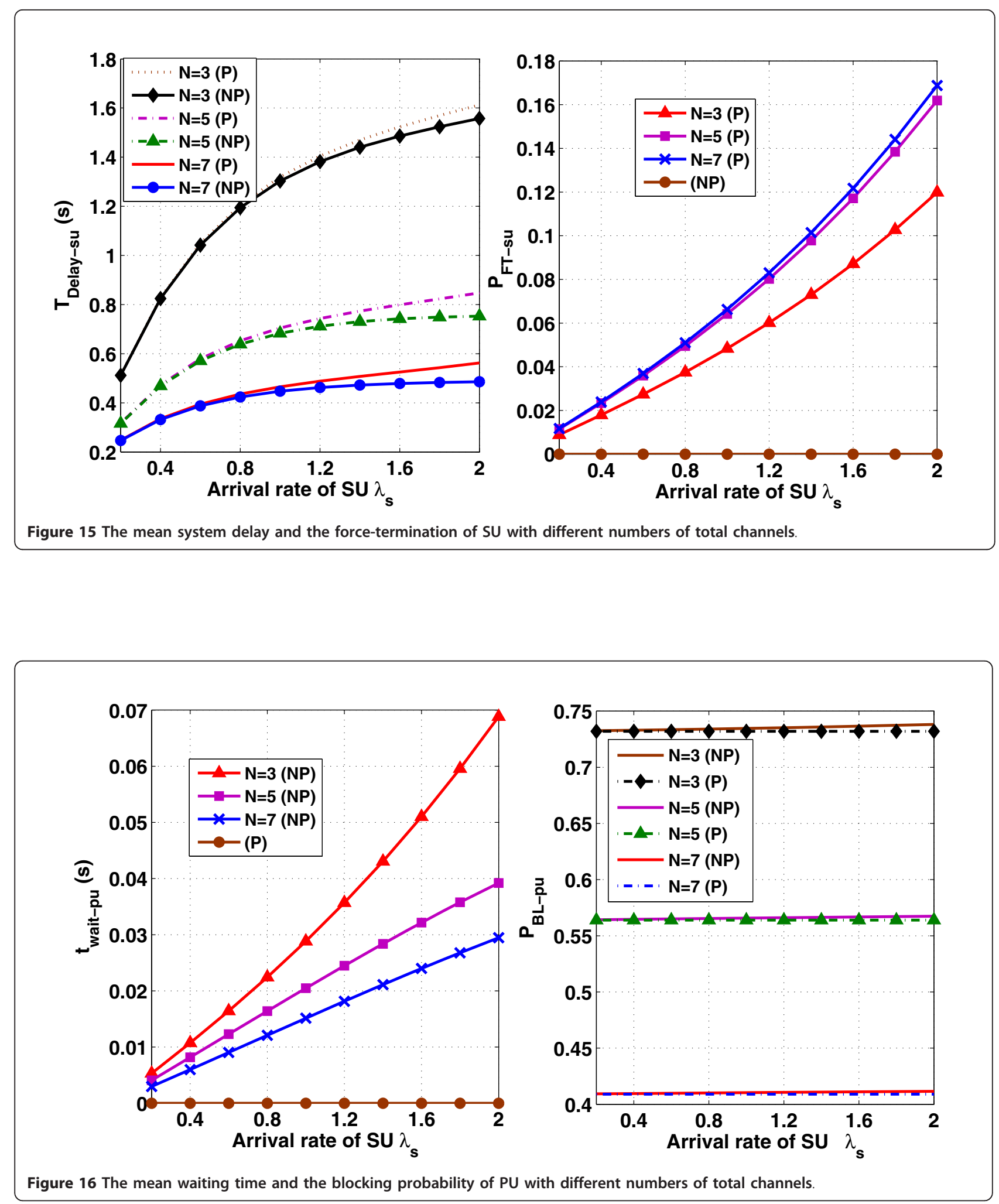
by setting the system parameters in designing spectrum leasing strategy will be an interesting topic for future study.

\section{Appendix}

\section{A. Complete algorithm of the preemptive mechanism}

For $i=0$ to $\mathrm{N}$

Calculate $P_{i}$ by using equation (1)

End For

For $i=0$ to $R$

Let $N-R=D, N-R+Q=E$

sub - A $\left\{\begin{array}{c}\text { For } k=0 \text { to } \min (N-R, N-i) \\ \text { If } k<\min (N-R, N-i) \\ \quad j=k \\ \text { Calculate } P_{i, j, \min (j, N-R)}^{\text {pre }} \text { by using equation (2) (3) } \\ \text { else } \\ \text { For } j=k \text { to } k+Q \\ \text { Calculate } P_{i, j, \min (j, N-R)}^{p y \text { using equation (2) (3) }} \text { End For } \\ \text { End If } \\ \text { End For }\end{array}\right.$

End For

For $i=R+1$ to $\mathrm{N}-1$

Let $N-i=D, N-i+Q=E$

Repeat sub - A

End For

$i=N, k=0$

For $j=0$ to $Q$

Calculate $P_{i, j, \min (j, N-R)}^{\text {pre }}$ by using equation (4) (5)

End For

\section{B. Complete algorithm of the NP mechanism}

For $i=0$ to $\mathrm{N}$

Calculate $P_{i}$ by using equation (1)

End For

For $i=0$ to $R$

Let $N-R=D, N-R+Q=E$

sub - A

End For

// calculate the non - queueing states with $i=R$

\section{Complement of simulation results}

(1) Different buffer sizes (Figures 13 and 14).

(2) Different numbers of total channels (Figures 15 and 16).

\section{Acknowledgements}

This study was supported by the National Basic Research Program under Grant No. 2009CB320402.

\section{Competing interests}

The authors declare that they have no competing interests.

Received: 13 April 2011 Accepted: 11 October 2011

Published: 11 October 2011
References

1. S Haykin, Cognitive radio: brain-empowered wireless communications. IEEE J Sel Areas Commun. 23(2), 201-220 (2005)

2. PK Tang, YH Chew, LC Ong, MK Haldar, Performance of secondary radios in spectrum sharing with prioritized primary access, in Proceedings of IEEE Conference on MILCOM 2006, Washington, DC, 1-7 (October 2006)

3. YR Kondareddy, N Andrewa, P Agrawal, On the capacity of secondary users in a cognitive radio network, in Proceedings of IEEE Sarnoff Symposium 2009, New Jersey, USA, 1-5 (March 2009)

4. SM Kannappa, M Saquib, Performance analysis of a cognitive network with dynamic spectrum assignment to secondary users, in Proceedings of IEEE International Conference on Communications (ICC) 2010, Ottawa, Canada, 1-5 (May 2010)

5. P Zhu, J Li, X Wang, Scheduling model for cognitive radio, in Proceedings of 3rd International Conference on Cognitive Radio Oriented Wireless Networks and Communications 2008, Singapore, 1-6 (May 2008)

6. S Sengupta, M Chatterjee, S Ganguly, An economic framework for spectrum allocation and service pricing with competitive wireless service providers, in Proceedings of 2nd IEEE International Symposium on New Frontiers in Dynamic Spectrum Access Networks 2007, Dublin, Ireland, 89-98 (April 2007)

7. MM Buddhikot, Understanding dynamic spectrum access: models, taxonomy and challenges, in Proceedings of 2nd IEEE International Symposium on New Frontiers in Dynamic Spectrum Access Networks 2007, Dublin, Ireland, 649-663 (April 2007)

8. K Hyoil, KG Shin, Optimal admission and eviction control of secondary users at Cognitive Radio HotSpots, in Proceedings of 6th Annual IEEE Communication Society Conference on Sensor, Mesh and Ad Hoc Communications and Networks, Rome, Italy, 1-9 (June 2009)

9. S Ghani, M Schwartz, A decomposition approximation for the analysis of voice/data integration. IEEE Trans Commun. 42(7), 2441-2452 (1994). doi:10.1109/26.297853

10. S Ghani, M Schwartz, A decomposition approximation for the performance evaluation of non-preemptive priority in GSM/GPRS, in Proceedings of 1st International Conference on Broadband Networks, California, USA, 459-469 (October 2004)

11. K Hyoil, KG Shin, Understanding Wi-Fi 2.0: from the economical perspective of wireless service providers. IEEE Wirel Commun. 17(4), 41-46 (2010)

12. J Jia, Q Zhang, Competitions and dynamics of duopoly wireless service providers in dynamic spectrum market, in Proceedings of ACM MobileHoc 2008, Hong Kong, 313-322 (May 2008)

13. D Soldani, M Li, R Cuny, QoS and QoE Management in UMTS Cellular System, (Wiley, New York, 2006)

doi:10.1186/1687-1499-2011-129

Cite this article as: Peipei et al:: Performance analysis of spectrum sharing mechanisms in cognitive radio networks. EURASIP Journal on Wireless Communications and Networking 2011 2011:129.

\section{Submit your manuscript to a SpringerOpen ${ }^{\mathcal{O}}$ journal and benefit from:}

- Convenient online submission

- Rigorous peer review

- Immediate publication on acceptance

- Open access: articles freely available online

- High visibility within the field

- Retaining the copyright to your article

Submit your next manuscript at $>$ springeropen.com 